\title{
Computer Aided Drug Design for finding a therapeutics for Dengue Virus Targets
}

\author{
A.Jainul Fathima, G.Murugaboopathi
}

\begin{abstract}
The dengue epidemic has taken aback the entire world today. It affects millions of people worldwide sometimes causing severe manifestation, affecting body metabolomics. It's caused by an arthropod-bornesingle-stranded RNA virus that has been distributed across the coastal regions of the globe with the advent of commercialization and trade. There is no effective treatment for dengue till date, but different forms of anti-viral vaccines are in the process of clinical trials for human use. Computational methods are being developed to unravel the viral transmission mechanics and evolution. Numerous networking models are being proposed to understand the phylogeny and inheritance pattern of the virus. Data models are projected in terms of mechanics or statistics to consider the distribution pattern of dengue in the future. This article talk about dengue virus targets at its genomics level.Several case scenario of applying CADD tools for finding the lead molecule for dengue targets were discussed. Advancement in dengue research with recent developments in computational methods were analyzed. The outcome of the present study suggested advancement in computational approaches may offer focused development of drugs for dengue.
\end{abstract}

Keywords : Anti-viral Research; CADD; Dengue NS2B/NS3 protease

\section{INTRODUCTION}

The Dengue outbreak, a major health concern today, hits nearly 2.5 billion people worldwide. It has infected more than 100 countries, causing 25000 deaths every year. It was first reported by Benjamin Rush in 1789 as the "bilious remitting fever" during the Philadelphia pandemic. With symptoms being flu-like it is caused by a RNA virus belonging to genus Flavivirus and the family Flaviviridiae affecting individuals of any age. The dengue infection may progress to spectrum of complication from mild dengue cases to fatal dengue hemorrhagic fever or dengue shock syndrome (DHF/DSS), if left untreated ${ }^{[1]}$. According to WHO, there has been a 30 fold upsurge worldwide in the rate of dengue between the years 1960 to 2018.Increased population growth, unplanned urbanization, global warming, lack of healthcare facilities, inefficient mosquito control, frequent air travels has been elucidated as some of the reasons for its increased prevalence ${ }^{[2][3]}$. Apart from this, dengue can be more predisposing in individuals with diabetes, bronchial asthma or polymorphisms in genes

Revised Manuscript Received on December 16, 2019.

* Correspondence Author

A. Jainul Fathima *, Department of Computer Science and Engineering, Kalasalingam Academy of Research and Education, Krishnankoil, India. Email:ajainulfathima@gmail.com.

G.Murugaboopathi, Department of Computer Science and Engineering, Kalasalingam Academy of Research and Education, Krishnankoil, India.. Email: gmurugaboopathi@ gmail.com like CTLA4, TGF beta, DC sign, vitamin D receptor etc. A cross link of physical, genetic and socio-economic factors come in to play to make an individual more susceptible to be affected by dengue. It inflicts not only severe health concerns but also escalates the socio-economic burden of the affected countries $\left[{ }^{4]}\right.$. A recent imperative taken on anti-viral drug discoveries and developing vaccine with effective management strategies has been able to downturn the percentage of dengue to a great extent. Recently a tetravalent vaccine has been licensed in several dengue affectedcountries; but its efficacy varies widely. So there remains a very strong urge to develop a uniform therapeutic modality to treat dengue. However, recent advances in Computer Aided Drug designing have come to the rescue, reducing the cost and time of performing wet lab experiments. Several compounds in the form of bromocriptine, ZINC92615064 has been found to be highly potent to treat dengue-likea virus ${ }^{[5]}$, targeting its translation machinery in the form of proteases, unraveling hopes to eradicate dengue completely.

DENV are arthropod bornesingle stranded virus with humans serving as the primary host and mosquitoes of the genus Aedes being the secondary host. There are five phylogenetically and antigenically distinct dengue viruses (DENV1-DENV5) and infection by one serotype confers immunity to that particular type and not the others ${ }^{[6][7]}$. The ancestor of this virus was believed to have emerged about 1000 years ago, but it was not until 1945, that the first two strains namely DENV1 and DENV2 were reported in Japan and Hawaii. However at the latter half of the $20^{\text {th }}$ century the transmission escalated following the spread of its principal vector, Aedes Aegypti in either Africa or Asia. Commercialization and business transport using ship vessels allowed for the introduction of the virus and the mosquito to coastal destinations around the world. Its spread along the urban and suburban regions with tropical and subtropical climates ${ }^{[8][9][10]}$. Human acquire the virus when bitten by an infected mosquito. Having an incubation period within 3-14 days the symptoms first appear in the form of muscular and joint pains, maculopapular rashes, bleeding gums and a positive tourniquet test result. Insight in to virus metabolism unmystify that the homeostatic process, autophagy pathway and ER stress mechanism to be altered in host body (humans) that enhances their further reproduction ${ }^{[11][12]}$. Serological mapping of the strains have shown a mass distribution of DENV1, DENV2 in the American regions and DENV2, DENV3 in the Asian regions. Regional Mapping has estimated about $75 \%$ of the global population being exposed to dengue in Asia-Pacific region putting in threat lives of 1.3 billion people. About 1.6 million cases were reported from America alone by the year 2010.Epidemic persists on regular 3-5 cycles throughout Asia and Africa. No comprehensive study or 


\section{Computer Aided Drug Design for finding a therapeutics for Dengue Virus Targets}

reports have been so obtained from Africa amidst the geographic spread of dengue worldwide. However, the recent outbreak data analysis performed by Amarasinghe et al in 2011 showed 34 African countries to be endemic in the dengue transmission. Due to high occurrence of malaria in the African countries the majority of "febrile illness" including dengue is often to be misdiagnosed as malaria surpassing the reports incidence. Croatia and France of Europe has reported cases of local transmission of dengue. with majority of Europe being dengue free. So, dengue is now endemic across 125 countries globally. The future of dengue predicts higher population estimates being affected by the deadly disease and one of the potential causes of it is the ability of virus to evolve and adapt to climatic and socio-economic changes of the human host populations. Genotypes modifications, with greater virulence are wiping out strains of lesser epidemiological impact, further assisting in its survival and duplication process ${ }^{[13]}$. Therefore effective strategies, projects should be undertaken to reduce the overall "Dengue burden" and reduce the mortality rates respectively.

\section{A. Dengue Drug Targets}

DENV is an enveloped single stranded positive sense RNA virus having a genome of approximately 10,700 nucleotides encoding 3411 amino acids ${ }^{[14][15]}$; that consists of structural proteins in the form of capsid, precursor membrane, envelope which are components of mature virus particle and seven non structural proteins that are present in affected cells. Graphical representation of the DENV genomic structure is represented in Fig.1. The open reading frame is flanked by two untranslated regions (5' and 3' UTR), where the 5' region contains a type 1 cap and the 3 ' end has a conserved stem loop structure ${ }^{[15]}$. The $5^{\prime}$ and $3^{\prime}$ UTR have complementary sequences that help them hybridize in order to initiate genome cyclization and RNA synthesis[16]. The first step in the viral transmission of a disease is the entry of virus into the host cell through receptor meditated endocytosis mostly within endosomes. With receptors being glycoprotein receptor, mannose like receptor, C-type lecithin like molecule (CLEC5A), the viral glycoprotein affects the host cell receptor binding and is an important target for humoral immunity ${ }^{[16][17]}$. Upon internalization, the acidic ph of the endosomes triggers a conformational change that induces the release of viral genome into the host cell. The replication complex accumulates along the cytosolic pockets and vesicles with the non-structural proteins serving the purpose of cleaving structural proteins, polyprotein processing and assisting in the process of viral replication ${ }^{[17][18][19]}$. The NS1 non-structural protein is conserved among most Flavivirus and is proposed to protect DENV from complement-dependent neutralization in solution, whereas the NS5 protein is responsible to assisting in the synthesizing of new viralRNA genomes ${ }^{[20][21]}$. Upon replication and proper packaging of the viruses, they are secreted in the host body followed by further entry in to host cell and the loop continues. The transmission loop of the DENV virus majorly depends on its vector Aedes aaegypti and to a lesser extent by Aedes albopictus. Once ingested by the mosquito, the viral replication takes place within its gut, which on its next blood meal is transferred to its primary host "The Humans". The viruses don't seem to affect the survival capacity of mosquitoes, but it however deteriorates the quality of life of an infected human, often having the potential to cause $\operatorname{death}^{[22][23]}$

\section{B. Importance of Non-Structural proteins in Dengue Virus}

Non-Structural proteins present in virus serves both canonical and non-canonical roles. Among the canonical roles most NSP's are associated to viral replication mechanism, where they interact with co-factors on interconnected lipid vesicles derived from endoplasmic reticulum forming the replication complex. Of them NS1 is a conserved glycosylated protein, where its dimeric form associates to transmembrame proteins NS4A and NS4B required for the activation of the replication complex. The hexameric forms interact with proteins of the complement system affecting the immune cellular response of the host.NS3 act as a protease, Rnase, nucleoside-5-triphosphatase in association with the co-factor NS2B .NS5 being the most conserved among flavivirus helps in capping, methylations and replication of the viral genome. The NS5 dimer associates with NS3 and NS2B that is necessary to initiate protein-protein and RNA-protein interactions required for polymerizationsThe $\mathrm{N}$-terminal domain of NS5 has distinct GTP and S-adenosylmethionine binding sites which further enhance and helps in the process of capping and RNA -synthesis. ${ }^{[24]}$ For the non-canonical roles the hexameric NS1 helps in circulating triglycerides, phospholipids and cholesterol. They were shown to have tropism in the liver when injected to mice followed by its internalization exhibiting the fact that NS1 helps in carrying lipids in the plasma of the dengue patients from the tissue to the liver. It has also been seen that antibodies produced against NS1 cross reacts with endothelial cells and platelets causing dehydration, plasma leakage and hypovolemic shock , followed by binding with coagulators like prothrombin and thrombrin in dengue patients. With respect to immunopathology-the expression of NS5 protein was shown to induce the secretion of IL- 8 and TNF-alpha that elevated the level of expression of adhesion molecules and induced endothelial cell permeability.NS5 is also associated to increased expression of cytokines RANTES that contribute to DENV pathogenesis by aggravating cytokine productivity. ${ }^{[25]}$

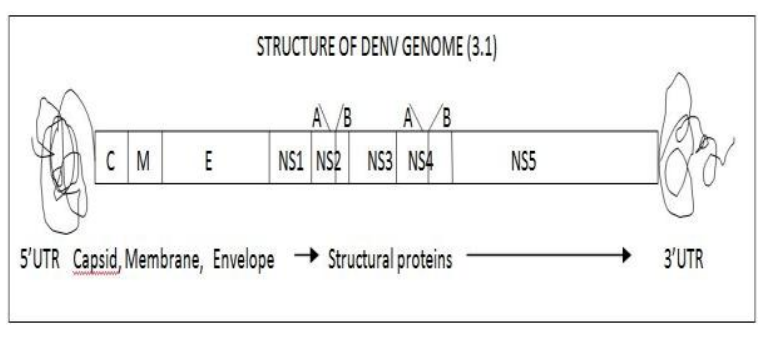

Fig 1 : Genomic Structure of Dengue Virus Genes

\section{REVIEW STATUS OF THERAPEUTICS FOR DENGUE}

The first anti-viral drug discovery was in the form of 5-iodo-2 desoxyuridine against herpes virus in the year 1959. The first antiviral drug available clinically was acyclovir by Getrude.B.Elion for which she was awarded the Nobel Prize in the year

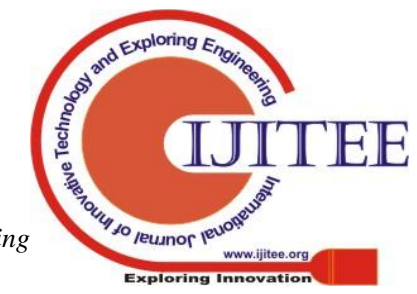


1988. With the advent of AIDS, Influenza and SARS pandemic the field of antiviral research widened allowing technical developments and therapeutic modalities Initially, infected cell assays were performed to come up with anti-viral treatments, where several compounds are tested in an infected cell culture system, for its efficacy. The ideal drug should have an excellent safety profile, should be an oral drug with a minimum dosage of 5 days duration as per as accordance with the Target Product Profile. Till date, 5 completed and published and 2 ongoing random clinical trials for anti-dengue agents have been carried out since the year 2000. Some of them being chloroquinone which is a 4-amino quinoline derivative ${ }^{[26]}$.It known to disrupt endosomal fusion and viral maturation. Predinisolone, a corticosteroid with anti-inflammatory properties. Some non randomized clinical trials have shown possibility of Predinisolone to treat dengue fever but controversies still persists. Balapiravir a 4' azidocytdine nucleoside analog served as a therapeutic agent against hepatitis $\mathrm{C}$ that belong to the same family Flavivirus that of the dengue virus. However prolonged exposure to it showed a toxic action. Ribavirin is a broad spectrum anti-viral drug that has RNA-polymerase inhibitory activity, Celgosivir that inhibits glycosylation of the viral coat proteins 'Ivermectin that inhibits host import nuclear receptors that is required for efficient viral replication and Ketotifen that promotes mast cell degranulation all shows high potential to treat dengue, however no statistically significant difference was seen between the control and treatment groups ${ }^{[27]}$. However plant extracts though a potent field in treating disease cannot be used in anti-viral therapy because of high toxicity, false positive results and cannot be modified to other forms of compound

To understand the pathophysiology of Dengue, labs are cultivating dengue in C36/36 mosquito cells or Vero African Green Monkey Kidney Cells,followed by infecting several human cell lines like Hela human cervical carcinoma cells , human foreskin fibroblasts, THP-1 myeloid cells . The level of dengue infectivitderivatives y can then be studied using methods such as plaque assay, qRT PCR, immunofluroscence and in-vitro report systems. Different agents in the form of RNA dependent RNA polymerase (NS5) inhibitors like N- sulfonylanthranilic acid derivatives were identified recently that binds to DENV NS5 at the site of entrance of the RNA tunnel and inhibits viral replication [28]. Nucleoside Analogues in the form of Balapiravr is in its phase II clinical trial , a cytidine analog that was initially developed for the treatment of HCV.Protease inhibitors in the form lof recombinant NS2B-NS3 protase retrocyclin 1 is deemed to se4ve as a potent DENV inhibitors. Other DENV inhibitors and proteases in the form of BP13944(a NS3 protease inhibitor), a-ketoamides (Ns2b-NS3 serine proteases), Quinoline containing compounds, Methyl transferase (NS5) inhibitors, Capsid inhibitors-ST148 have been recently develop and is in the process of fclinical trials.Host modulators that uses or targets the host machinery that is required for the viral cell replication has been brought to the limelight recently as an effective strategy to treat dengue. The different modulators being Ribavarin, Mycophenolic acid,alpha-glucosidase inhibitors, lovastatin, heparin and heparan sulfate, Ivermectin, Pentoxifylline,Chloroquinone, RNA and Morpholinos. They mostly act by inhibiting inosine monophosphate dehydrogenase that results in GTP pool depletion or IMP dehydrogenases. RNA interference or siRNA delivery through the use of dendritic cell targeting peptide against a conserved sequence of virus has been shown to effectively suppress DENV replication in macrophages and monocytes. A recently synthesized focused library of 1,2 -benzisothiazol-3(2H)-one-1,3,4-oxadiazole has been created which were found to inhibit DENV NS2B/NS3 through competitive inhibition. ${ }^{[29,30]}$ So, there are multiple directions to antiviral drug design advancing, but major challenges include development and identification of a compound that is validated in-vivo, with a high-safety profile, able to be active beyond the earliest hours of infection and most importantly inexpensive and public-usable .[31] $^{\text {no }}$

\section{REVIEW ON COMPUTER AIDED DRUG DESIGN TOOLS FOR DENGUE}

Knowledge based methods differ from normal wet lab cell screening process is that they use screening systems at the molecular and atomic levels that reduces cost and increase efficiency. Computer Aided Structural Activity relationship (SAR) ${ }^{[32]}$ or In-Silico drug designing facilitates such a cause that helps in designing drugs setting drug resistance as a criterion. There are a wide number of software's like grid computing, windows based PBPK/PD modeling software's, PKUDDS ${ }^{[33]}$ for structure based drug design etc. Different methodologies are used to develop drug any clinical stage of a disease such as Homology Modeling which involves comparative modeling of protein based on the atomic resolution model of a target protein and a related homologous protein ${ }^{[34]}$ Molecular docking is another such technique which foretells the favored orientation of one molecule to a second, forming a stable complex and is mostly used for ligand-receptor binding studies. Virtual high-throughput screening evaluates large libraries of compounds for their capacity to bind to specific target molecules ${ }^{[35] .}$ Quantitative structure activity (QSAR) relationships and hologram quantitative structural activity relationship (HSAR)[36] that is used to envisage relationships of structure and/or property descriptor of compounds with their biological activities. Some of the other used technique includes 3D pharcamophore mapping, microarray analysis, Monte Carlo stimulation, Molecular dyanamic stimulation etc ${ }^{[37][38]}$

\section{A. NS2B/NS3 Protease Target Specific inhibitors as Anti-Dengue Compounds using pharcamophore Mapping Studies}

Research has shown that the activation and potential of non structural viral protein NS3 depends upon its interface with co-factor NS2B. The NS3-NS2B complex helps in cleaving viral protein in order to make them functional. Disruption in this interaction is reported to inhibit viral replication and serves an effective target to anti-dengue therapy. Therefore the NS3-NS2B proteases three dimensional structure was retrieved using Protein Data Bank followed by virtual screening, semi-flexible docking and MD stimulations. On a potential pharcamophore search, Bromocriptine was retrieved from the ZINC database and was found to be a potential 
inhibitor.40, 000 bromocriptine like compounds was found using virtual screening and saved. Molecular docking was performed and a total of 39, 9722 compounds were successfully docked on NS2B-NS3 protease drug with binding energies between $(-6.35)$ to $(-16.45) \mathrm{kcal}$. The ZINC92615064 compound was found to be the best binder to the proteases keeping in mind its toxic properties too. The pharmacological properties assessed and MD stimulations were performed, for both Bromocriptine and ZINC9261504 While Bromocriptine was shown to have 22 points of contact with the NS2B-NS3 complex, ZINC9261504 established 25 contacts, showing to have a better binding capacity $^{[39] .}$

\section{B. Lead Optimization Studies using ZINC92615064 towards finding NS2B/NS3 drug specific inhibitors}

The identified lead compound ZINC92615064 was made to undergo scaffold hopping based novel library generation. A total of 16,847 compounds novel design compounds library was generated. Virtual screening was performed for this compounds using PyRx software where Template No 3718 compound was recognized to be successfully binding with the template and having a binding energy of (-9.2) $\mathrm{kcal} / \mathrm{mol}$ along with ADMET parameters in a permissible range. The template form 3718 has exhibited much improved direct bonding with the residues at the active site of the target along with some Vander Wals interactions that further plays a crucial role in complex formation. Molecular docking simulations and MD stimulations were performed to further validate the compound pharmacologically. There were 27 contacts found in between the compound 3718 and the protease target compared to only 25 bonds with the compounds ZINC92615064, thus having a better binding potential ${ }^{[39][5]}$.

\section{In-Silico Drug Designing Studies on Dengue Virus NS1 protein}

The compounds used for drug designing for this non-structural protein is the phytochemicals obtained from Dragon fruitseed. The dragon fruit seed belongs to cactacea family and they are rich in compounds that have been previously reported to treat dengue, neurodegenerative disorder and hypercholestremia.A group of 11 compounds were selected for in-silico study that included 7,10,13-hexadecatrienoic acid, 9,12,15-octadecatrienoic acid, 9,12-octadecadienoic acid, 9,17-octadecadienal, methyl-8,11,14-heptadecatrienoate, n-hexadecanoic acid, nonanoic acid, octadecanoic acid, phytol, S-(-)-1,2,4-butanetriol and tetradecanoic acid . Ligands were constructed using ChemSketch and optimized by the addition of hydrogen bonds followed by docking studies. Among them three compounds namely 7, 10, 13-hexadecatrienoic acid (Compound A), 9, 12, 15-octadecatrienoic acid (Compound B), 9, 12-octadecadienoic acid (Compound C) showed lower ligand binding energy and hence greater affinity for the NS1 protein ${ }^{[40,41]}$. However, proper in-silico studies is needed to understand the structure of the active sites for the NS1 protein, followed by wet lab experiments to validate the results from the in-silico studies for the above mentioned compounds.

\section{In-Silico Studies of Dengue Viral Proteins with selected Coriandrum Sativum $L$ leaf extracts}

Coriandrum Sativum plants are considered to be an important source of medicine and drugs used for the treatment of Alzheimer's, cancer, dysentery, insomnia, menstrual disorders etc. The hydrodistilled extracts of plants showed the presence of Dodecanal (Synonym: Lauraldehyde), E-2- Dodecanol (Synonym: Dodecan-2-ol), Decanal (synonym: Decylaldehyde) and E-2- Decenol (synonym: Trans-2-Decen-1-ol). The structure of all the five non-structural proteins was obtained from Protein Data Bank while that of the extracts from ChemSketch. Docking studies were performed and it was seen that the compound E-2-Dodecanol had the least ligand binding energy, hence greater binding affinity, serving as a potential drug target. Several in-vitro analysis have been performed to test the efficacy of the compound, however further in-silico studies are required to unravel the structure and the amino acid residues of the active site of the compound and its association with the proteins ${ }^{[42] \text {. }}$

\section{E. In-Silico Study on Inhibition of NS5 protein on Dengue Virus.}

The NS5 non-structural protein in flavivirus is highly conserved and mostly associated to capping mechanism in flavivirus. The sequences for viral proteins were retrieved using Protein Database followed by BLAST-P to check sequence similarity .The model for viral proteins were created using SWISS model server followed by uses of databases like LIGAND EXPO, -IGEMDOCK and HEX SERVER. 7-METHYL-GUANOSINE-5'TRIPHOSPHATE-5'-(2'-O-METHYL)- GUANOSINE and B-OCTYLGLUCOSIDE were found to have binding energy of -123.2 and -84.61 respectively for envelope protein and NS5 protein. Their binding causes functional disabilities to the protein machinery thus inhibiting viral replication process and serving as possible molecules in further drug development. $^{[43]}$

\section{F. Other Computational Studies in Relation to Dengue Virus}

The genome of the dengue virus shows the presence of several cis acting elements of which a 719 nucleotide subgenomic minigenome is an important sequence and template for the process of translation Probing analysis showed hybridization between cis acting elements at the 5'-3' end with the formation of dumbbell and hairloop like structures. Disrupting the hybridized interaction via flipping mutation has previously shown to attenuate DENV replication. Computer Modeling studies indicated that the hybridization motif might function as an autonomous regulatory element and depend on the sequence and composition of 5' cyclization region. 


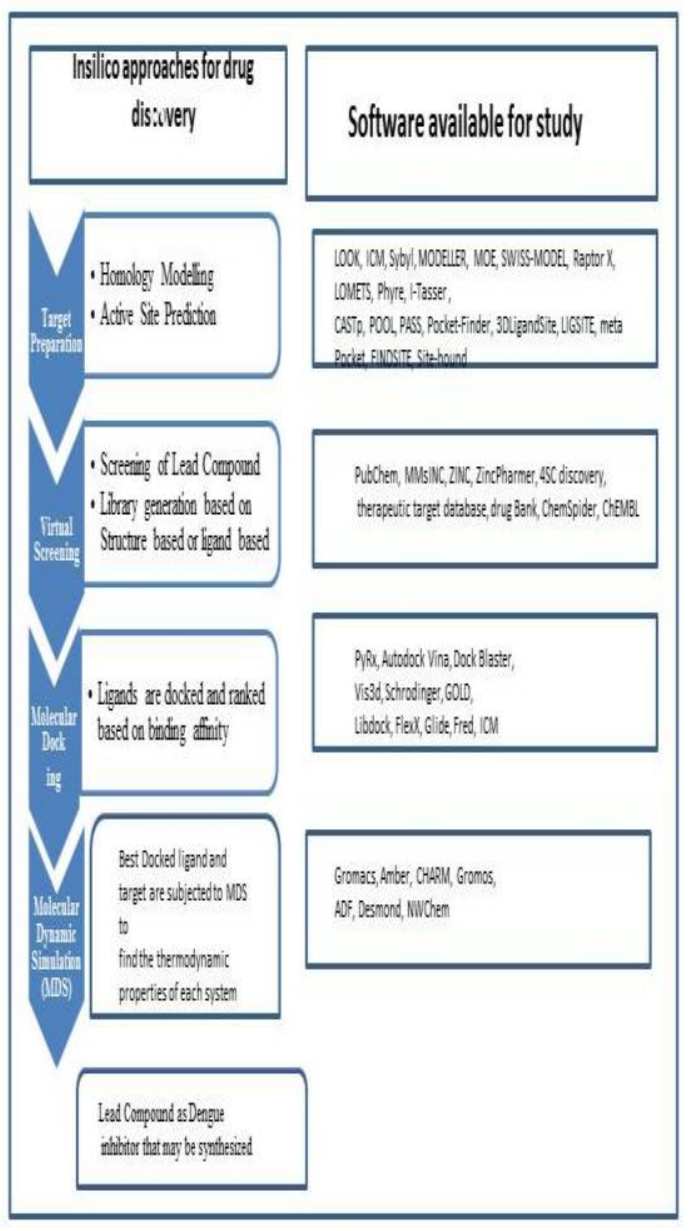

Fig 2: Route Map design of dengue virus Inhibitors

\section{ROUTE MAP TOWARDS THE COMPUTATIONAL DESIGN OF DENGUE INHIBITORS}

The Computer Aided Drug Design Approach is classified in to Structure based and ligand based approach. Various computational steps involved in in-silico approach are target selection, Lead Identification, Molecular Docking and Molecular Dynamic Simulation Studies. Many Software tools are available for each stages of study and are clearly shown in Figure 2. Machine Learning Algorithms like Support Vector Machine (SVM), Deep Learning (DL), Random Forest (RF) can be applied in all stages of drug development process. Application of these algorithms will improvise the accuracy in prediction of targets and screening of drugs. In Dengue research machine learning algorithms are applied to develop a forecasting model. These models are used by the government to find the scheme needed to strengthen dengue control

\section{CONCLUSION}

Urbanization trends and human movement patterns have been thought as the key potential drivers for the dengue endemicity over the last 70 years. Since its vector resides in a pre-domestic urban environment, the increasing trends of commercialization would have a significant effect on its distribution pattern. The use of bioinformatics tools at such an ever demanding scenario to develop molecular modeling programs and high performance computing for drug

designing is of great importance. One of the most important strategies in in-silico work being virtual screening of databases which can identify new potential protein ligands as an effective target to treat dengue. Using protein databases to retrieve the structure of dengue virus protein followed by virtual screening helps in identifying low molecular weight molecules that may have a proteolytic activity inhibiting the process of viral replication. In the future, the most important tendency in computational drug discovery would be the integration of computational chemistry and biology together with chemoinformatics and bioinformatics which would result in the emergence of a new field like pharmacoinformatics making drug discovery an easier and an efficient process. The outcome of the present study suggested advancement in computational approaches may offer targeted and cost-efficient models to develop new drugs for dengue target.

\section{REFERENCES}

[1] A. F. L. T. Argolo et al., "Prevalence and incidence of dengue virus and antibody placental transfer during late pregnancy in central Brazil,", 2013.

[2] S. M. A. A. A. B. Shamimul Hasan, Sami Faisal Jamdar, Munther Alalowi, "Dengue virus: A global human threat: Review of literature," J Int Soc Prev Community Dent., pp. 1-6, 2016.

[3] M. D. Kashinkunti, "A Study of Clinical Profile of Dengue Fever in a Tertiary Care Teaching Hospital," Sch. J. Appl. Med. Sci. Sch. J. App. Med. Sci, vol. 1, no. 4, pp. 280-282, 2013.

[4] V. Srinivas and V. R. Srinivas, "Dengue Fever: a Review Article," J. Evol. Med. Dent. Sci., vol. 4, no. 29, pp. 5048-5058, 2015.

[5] M. Gurusamy and J. Fathima, "Lead optimization studies towards finding NS2B/NS3 protease target-specific inhibitors as potentia anti-dengue drug-like compounds," Curr. Drug Discov. Technol., vol. 15 , pp. 1-8, 2018.

[6] G. Wilder-Smith A, Ooi EE, Vasudevan SG, "Update on dengue epidemiology, virus evolution, antiviral drugs, and vaccine development," Curr Infect Dis Rep., 2010.

[7] R.-H. R., "Microevolution and virulence of dengue viruses. Adv Virus Res.," 2003.

[8] "Comprehensive Guidelines for Prevention and Control of Dengue and Dengue Haemorrhagic Fever, Revised and Expanded Edition," 2011.

[9] G. D. Wilder-Smith A, "Geographic expansion of dengue: the impact of international travel.," 2008.

[10] G. DJ., "Epidemic dengue/dengue hemorrhagic fever as a public health, social and economic problem in the 21 st century," Trend Microbiol, 2002.

[11] "No Title." [Online]. Available: https://www.betterhealth.vic.gov.au/health/conditionsandtreatments/ Dengue-virus-disease?viewAsPdf=true.

[12] "No Title." [Online]. Available https://wwwnc.cdc.gov/travel/yellowbook/2016/infectious-diseases-r elated-to-travel/dengue.

[13] and S. I. H. Jane P. Messina, Oliver J. Brady, "Global spread of dengue virus types: mapping the 70 year history," 2014.

[14] "WHO. Geneva: WHO; 1997. Dengue haemorrhagic fever. Diagnosis, treatment, prevention and control;",

[15] D. A. Pang X, Zhang M, "Development of dengue virus replicons expressing HIV-1 gp120 and other heterologous genes: a potential future tool for dual vaccination against dengue virus and HIV.e," $B M C$ Microbio, 2001.

[16] D. A. Pang X, Zhang M, "Development of Dengue virus type 2 replicons capable of prolonged expression in host cells," $B M C$ Microbio, 2001.

[17] G. A. Alvarez DE, Lodeiro MF, Filomatori CV, Fucito S, Mondotte JA, "Structural and functional analysis of dengue virus RNA.," Novartis Found Symp, 2006. 


\section{Computer Aided Drug Design for finding a therapeutics for Dengue Virus Targets}

[18] L. M, "Flavivirus premembrane protein cleavage and spike heterodimer secretion require the function of the viral proteinase NS3," 1997.

[19] K. A. K. Westaway EG, Mackenzie JM, "Kunjin RNA replication and applications of Kunjin replicons. Adv Virus Res," 2003.

[20] et al. Chen Y, Maguire T, Hileman RE, Fromm JR, Esko JD, Linhardt RJ, "Dengue virus infectivity depends on envelope protein binding to target cell heparan sulfate.," Nat Med, 1997.

[21] L. C. Pethel M, Falgout B, "Mutational analysis of the octapeptide sequence motif at the NS1-NS2A cleavage junction of dengue type 4 virus.," 1992.

[22] et al. Miller JL, de Wet BJ, Martinez-Pomares L, Radcliffe CM, Dwek RA, Rudd PM, "The mannose receptor mediates dengue virus infection of macrophages. PLoS Pathog.," 2008.

[23] B. R. Welsch S, Miller S, Romero-Brey I, Merz A, Bleck CK, Walther P, Fuller SD, Antony C, Krijnse-Locker J, "Sites, Composition and three-dimensional architecture of the dengue virus replication and assembly," 2009.

[24] P. R. Boonyasuppayakorn S, "Construction of plasmid, bacterial expression, purification, and assay of dengue virus type 2 NS5 methyltransferase. Methods in molecular biology (Clifton, N.J.).," 2014.

[25] G. M. B. and A. T. D. P. * Julianna D. Zeidler, Lorena O Fernandes-Siqueira, "Non-Canonical Roles of Dengue Virus Non-Structural Proteins."

[26] B. Canard, "Antiviral Research and Development Against Dengue Virus.,"WHO Rep., pp. 1-101, 2011.

[27] R. G. Jenny G. Low and and A. S. Subhash G. Vasudevan, "Dengue Antiviral Development: A Continuing Journey," 2018.

[28] A. Burgess, K. Shah, O. Hough, and K. Hynynen, "HHS Public Access," vol. 15, no. 5, pp. 477-491, 2016.

[29] U. T. and C. Thisyakorn, "Latest developments and future directions in dengue vaccines," 2014.

[30] A. K. Lai H, Dou D, Aravapalli S, Teramoto T, Lushington GH, Mwania TM and G. W. Eichhorn DM, Padmanabhan R, "Design, synthesis and characterization of novel 1,2-benzisothiazol-3(2H)-one and 1,3,4-oxadiazole hybrid derivatives: potent inhibitors of Dengue and West Nile virus NS2B/NS3 proteases. Bioorganic \&amp; medicinal chemistry," 2012.

[31] F. D. and C. RT2, "Review of Current Dengue Treatment and Therapeutics in Development," 2014.

[32] Z. P. Kaczanowski S, "No Title," Why similar protein Seq. Encode similar three M Dimens. Struct. Theor. Chem. Accounts, 2010.

[33] S. AK., "Molecular modelling and drug design.2nd edition (Chennai), MB publisher," 2008.

[34] L. AJM., "Introduction to Bioinformatics," Oxford Univ. Press inc. New York, no. 2002.

[35] M. M. Walters WP, Stahl MT, "Virtual screening - an overview. Drug.Discov. Today," 1998.

[36] J. H. Suh M, Park S, "Comparison of QSAR Methods (CoMFA, CoMSIA, HQSAR) of Anticancer 1-N-Substituted Imidazoquinoline-4,9-dione Derivatives. Bull. Korean Chem. Soc," 2002.

[37] W. J. Meyer EF, Swanson SM, "Molecular modeling and drug design. PharmacolTher.," 2000.

[38] T. D. Allen MP, “Computer Simulation of Liquids, Oxford Science Publications, Oxford, UK," 1989.

[39] A. J. Fathima, G. Murugaboopathi, and P. Selvam, "Pharmacophore Mapping of Ligand Based Virtual Screening, Molecular Docking and Molecular Dynamic Simulation Studies for Finding Potent NS2B/NS3 Protease Inhibitors as Potential Anti-dengue Drug Compounds," Curr. Bioinform., vol. 13, no. 6, pp. 606-616, 2018.

[40] M. V. Garisekurthi Satheesh, Nagu P. Prabhu, "3D Modeling of Dengue Virus NS4B and Chikungunya Virus nsP4: Identification of a Common Drug Target and Designing a Single Antiviral Inhibitor," 2014.

[41] "IN SILICO DRUG DESIGNING STUDIES ON DENGUE VIRUS NS1 PROTEIN." [Online]. Available: https://www.pharmatutor.org/articles/in-silico-drug-designing-studies -on-dengue-virus-ns1-protein.

[42] W. Journal and O. F. Pharmaceutical, "IN SILICO STUDIES ON DENGUE AND RHINO VIRAL STRUCTURAL PROTEINS," vol. 4, no. 12, pp. 281-286, 2018.
[43] S. Raikar et al., "In silico Study on Inhibition of NS5 Protein of Dengue Virus," vol. 4, no. 2, pp. 28-43, 2017.

\section{AUTHORS PROFILE}

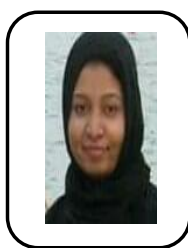

A. JainulFathima received her B.Tech. degree in Information Technology from Anna University- Chennai in 2007and M.Tech degree in Computer Science and Engineering from Anna University - Tirunelveli in 2009 . She has 3 years of teaching experience. She is currently pursuing Ph.D. degree in Kalasalingam Academy of Research and Education, Krishnankoil. Her Research area includes Big data analytics, Computational Drug discovery, andBioinformatics.She is a Life Member of the Indian Society for Technical Education (ISTE).

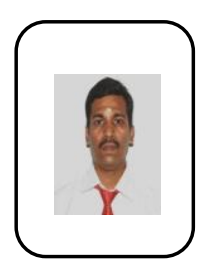

Dr.G.Murugaboopathi received the Undergraduate Degree in Computer Science and Engineering from Madurai Kamaraj University in 2000, the Post Graduate degree in Digital Communication and Network from Madurai Kamaraj University in 2002 and Ph.D in Computer Science and Engineering at Bharath University, Chennai. He has more than 45 publications in National, International Conference and International Journal proceedings. He has more than 15 years of teaching experience. His areas of interest include Wireless Sensor Networks, Bioinformatics.Mobile Communication, Mobile Adhoc Networks, Mobile Computing, Cloud Computing, Network Security, Network and Data Security, Cryptography and Network security. He is currently working as an Associate Professor in the Department of Computer Science and Engineering at Kalasalingam Academy of Research and Education, Tamil Nadu,India 